\title{
Successful Reposition of Prolapsed Silicone Tube Using Hole and Lacrimal Probe Method
}

\author{
Zee Yoon Byun ${ }^{1}$, Bo Ram Lee, Sung Chul Kim ${ }^{1,2}$ \\ ${ }^{1}$ Han Gil Eye Hospital, Incheon, Korea \\ ${ }^{2}$ Department of Ophthalmology, Catholic Kwandong University College of Medicine, Incheon, Korea
}

\begin{abstract}
Purpose: Silicone tube intubation is the most common method for treatment of nasolacrimal drainage disorder or repair of lacerated canaliculus. However, some cases are prolapsed earlier than expected, subsequently affecting the success rate of silicone tube intubation. The author introduced a successful reposition of the prolapsed silicone tube.

Methods: This study is a retrospective case series of 11 patients who were treated with reposition of prolapsed silicone tube using hole and lacrimal probe method. In all cases, the tubes were prolapsed within 2 months after the first surgery due to careless rubbing of the medial canthus by the patient. In all 11 cases, the development of a biofilm on tube was not observed at the time when the tube was prolapsed. All patients underwent immediate reposition of prolapsed tube without local anesthesia in the outpatient operating room on the day they visited.

Results: In all 11 cases, the prolapsed silicone tube was repositioned without any complications using this hole and lacrimal probe method. At 6-month follow-up examination, all patients presented with a well-positioned silicone tube up to the day of extubation without replacing it with a new silicone tube.

Conclusions: This hole and lacrimal probe method is effective for the repositioning of prolapsed silicone tube after bicanalicular intubation of lacrimal system. This procedure includes easy performance in a short time and without local anesthesia in the outpatient operating room.
\end{abstract}

Key Words: Hole, Lacrimal probe, Prolapsed silicone tube

Silicone tube intubation is the primary treatment for treatment of nasolacrimal drainage disorder. The indications for silicone tube intubation include punctal or lacrimal canalicular and nasolacrimal duct stenosis associated with allergic conjunctivitis, recurrent viral conjunctivitis, or anti-cancer medication $[1,2]$. The tube is usually re-

Received: March 19, 2021 Final revision: April 26, 2021

Accepted: April 27, 2021

Corresponding Author: Sung Chul Kim, MD. HanGil Eye Hospital, 35 Bupyeong-daero, Bupyeong-gu, Incheon 21388, Korea. Tel: 82-32-5033322, Fax: 82-32-503-0801, E-mail: 120301@hangileye.co.kr moved from the duct 6 months after intubation. Many factors affect the success rate of silicone tube intubation, such as biofilm-related infection, early silicone tube prolapse, or the preoperative condition of the lacrimal duct $[3,4]$. The cause of early silicone tube prolapse can be a surgeon-related factor such as inappropriate length of the silicone tube, but is more often to do with careless rubbing by the patient immediately after surgery. Therefore, including in some congenital nasolacrimal duct obstruction cases, ST fixation at the nasal mucosa using 6-0 Prolene or silicone sponge technique can be used to prevent early tube prolapse [5,6]. Recent studies by Chu et al. [7] and He et al. [8] 
describe methods that can be used to reposition the prolapsed silicone tube using a suture-probe or a memory wire probe, respectively. Herein, we present a new, effective hole and lacrimal probe method for repositioning the prolapsed silicone tube using lachrymal probe, dropshaped tip, size 0000-000, stainless steel (D-110S; Cilita, Ryazan, Russia) without local anesthesia.

\section{Materials and Methods}

The analysis was performed in adherence with the Declaration of Helsinki and after approval from the institutional review board and ethics committee of the HanGil Eye Hospital in Incheon, Korea. Because this study is a retrospective analysis of clinical data, we submitted a reason for exemption in written informed consent to institutional review board. From January 2019 to February 2021, all patients had been treated with bicanalicular silicone tube intubation for treatment of stenosis of punctum in four cases, stenosis of the punctum and canaliculus in two cases, stenosis of the punctum and nasolacrimal duct in three cases, and stenosis of canaliculus and nasolacrimal duct in two case. And 10 patients were decided to have the conjunctivoplastic surgery for the treatment of conjunctivochalasis. All patients enrolled in this study demonstrated prolapse of silicone tube at medial canthus but the tube was not visible in the inferior meatus under nasal endoscopy and thus could not be repositioned through nasal cavity in HanGil Eye Hospital. The tube dislocation occurred 1 day to 8 weeks after the first surgery. The cause of silicone tube prolapse was rubbing by the patients after initial intubation.

The procedure uses lachrymal probe, drop-shaped tip, size 0000-000, stainless steel (D-110S, Cilita). All patients underwent immediate reposition of prolapsed silicone tube using our new method without local anesthesia in the outpatient operating room.

First, the prolapsed silicone tube is held in a half circle and cut obliquely using Vannas scissors, not cutting completely (Fig. 1A). Subsequently, a small semicircular hole is created in the silicone tube (Fig. 1B). The probe is inserted
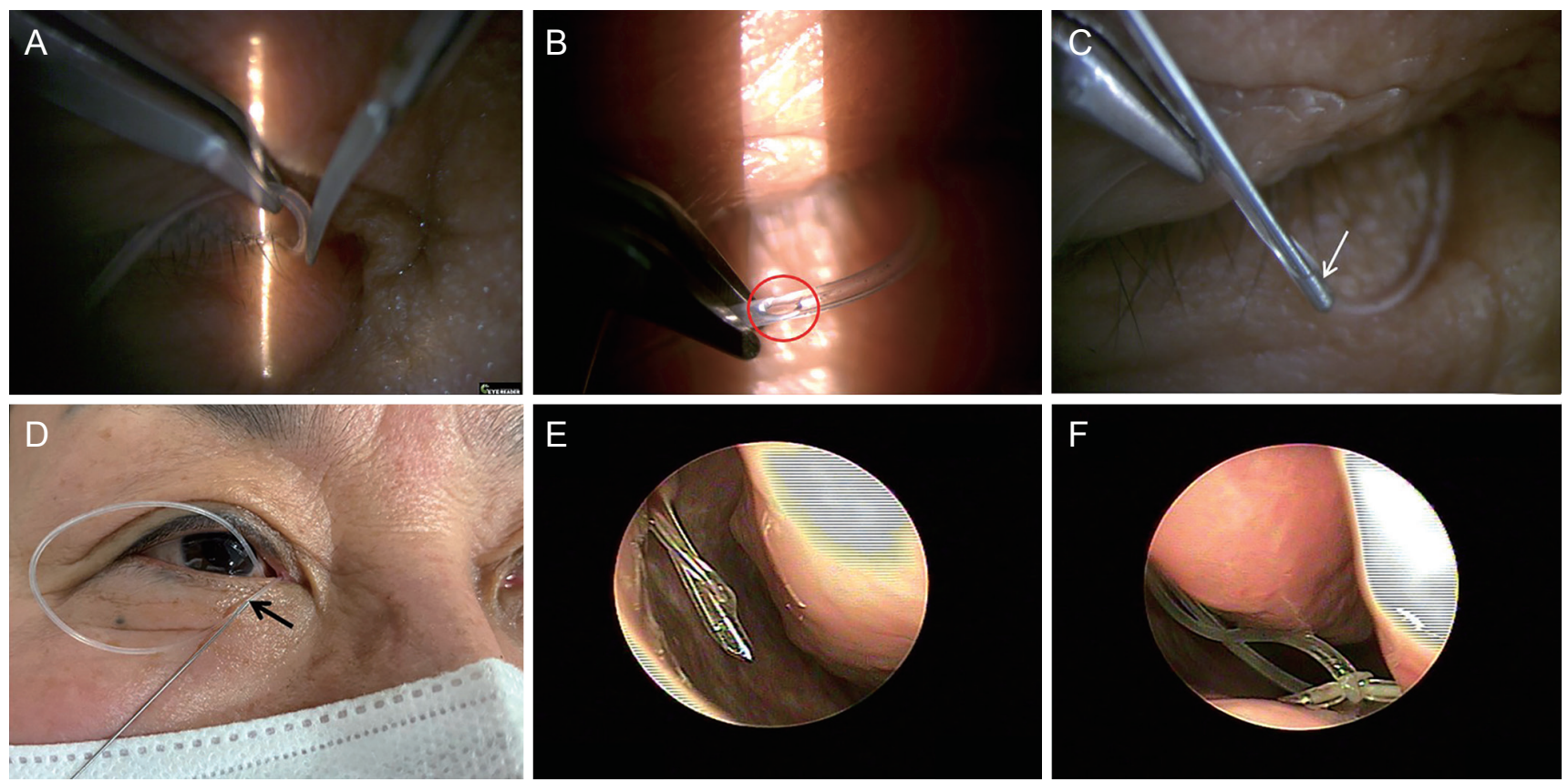

Fig. 1. The surgical procedures. (A) The prolapsed silicone tube is held in a half circle and cut obliquely using Vannas scissors, not cutting completely. (B) Subsequently, a small semicircular hole is created in the silicone tube (red circle). (C) The probe is inserted into the hole in the silicone tube (white arrow). (D) After proparacaine eye drops are applied, the probe already inserted through the hole (black arrow) into the silicone tube is carefully inserted into the Hasner's valve, as in the first operation. (E) The probe in the silicone tube can be seen from Hasner's valve under nasal endoscopy. (F) Finally, after the silicone tube from Hasner's valve is caught by Ritleng forceps, the probe is pulled back out from the upper punctum, with the result that the prolapsed silicone tube is repositioned. The tie portion of the silicone tube is properly repositioned in the inferior meatus using Bayonet dressing forceps. Informed consent for publication of the clinical images was obtained from the patient. 

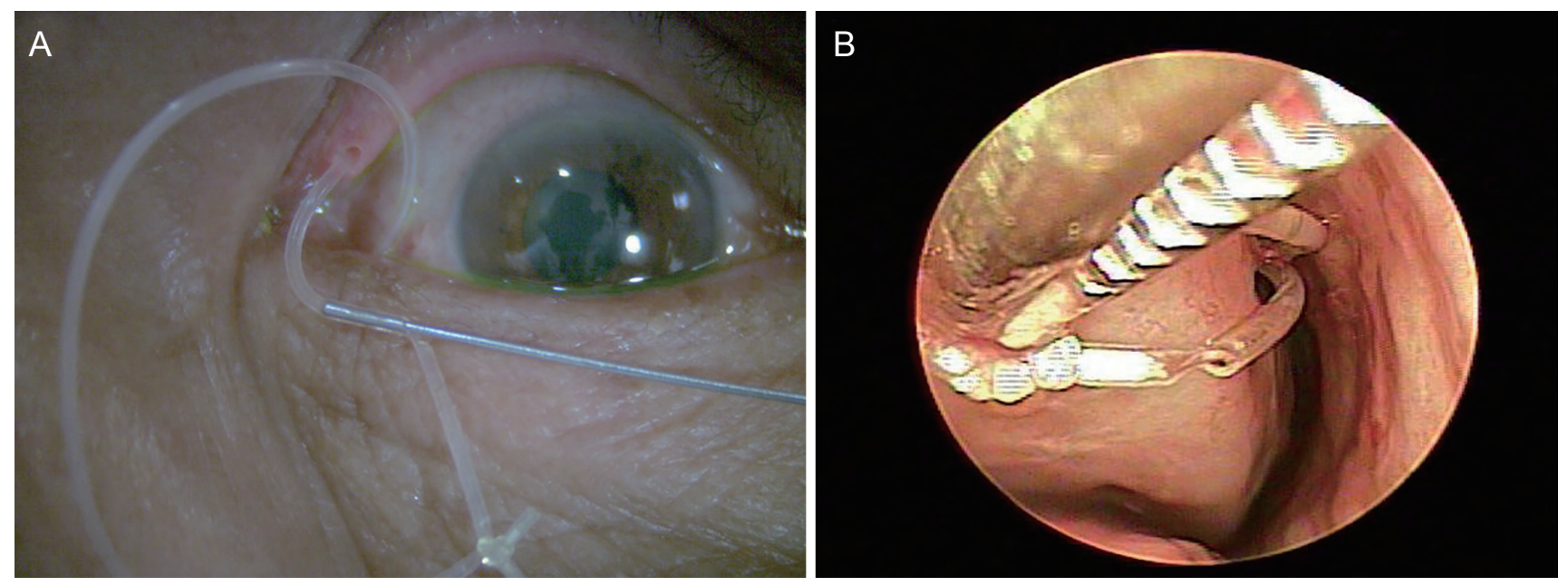

Fig. 2. The surgical procedures. (A) If the knot of the prolapsed tube is seen outside, it is important to make the hole close to the knot and insert the probe in the opposite direction of the knot into the hole to be allowed to enter the punctum first and then the knot to fellow through the punctum. (B) At the 3-month follow-up, the silicone tube is well positioned without break around the hole on nasal endoscopic examination. Informed consent for publication of the clinical images was obtained from the patient.

into the hole in the silicone tube (Fig. 1C). After proparacaine eye drops are applied, the probe already inserted through the hole into the silicone tube is carefully inserted into the Hasner's valve, as in the first operation (Fig. 1D). The probe in the silicone tube can be seen from Hasner's valve under nasal endoscopy (Fig. 1E). Finally, after the silicone tube from Hasner's valve is caught by Ritleng forceps, the probe is pulled back out from the upper punctum, with the result that the prolapsed silicone tube is well repositioned (Fig. 1F). The knot portion of the silicone tube is properly repositioned in the inferior meatus using Bayonet dressing forceps.

If the knot of the prolapsed silicone tube could be seen outside, it is important to make the hole close to the knot and insert probe in the opposite direction of the knot into the hole so that the probe can be allowed to enter the punctum first and then the knot to fellow through the punctum (Fig. 2A). The next procedure is the same as above mentioned. At the 3-month follow-up, the silicone tube was well positioned without break around the hole on nasal endoscopic examination (Fig. 2B).

\section{Results}

In all patients (five males and six females), the prolapsed silicone tube was successfully repositioned using this hole and lacrimal probe method without any complications. The mean age of all patients was 61.4 years (range, 14-82 years). The tube was never completely cut or broken during the insertion of the lacrimal probe into the hole made in the tube.

After silicone tube reposition, oral antibiotics for 3 days and antibiotic eye drops for 2 weeks were prescribed. Until extubation, all patients visited the hospital every month to check the condition of the tube using a nasal endoscope. At 6-month follow-up examination after initial intubation, all the patients presented with a well-positioned silicone tube without break around the hole on nasal endoscopic examination.

\section{Discussion}

Repositioning a prolapsed silicone tube which is not visible at the inferior meatus is very complicated. A prolapsed silicone tube after dacrycystorhinostomy surgery may be repositioned more simply, as the tube is easily visible through the rhinostomy site [9]. When a prolapsed tube is hard to reposition it may be removed at an early stage, resulting in lower success rate for nasolacrimal duct stenosis $[10,11]$. Chu et al. [7] reported that repositioning of the dislocated silicone tube was successful using suture-probe technique. He et al. [8] introduced a technique that uses memory wire probe and nylon to make a suture knot in order to capture the dislocated silicone tube. The above 
methods were both performed under local anesthesia and required special probes. Herein, we present a new technique that uses a Hole and lacrimal probe. A small hole is made to the prolapsed tube using Vannas scissors, and then after the probe is inserted into the hole, the engaged probe with the silicone tube is passed through the lacrimal sac and nasolacrimal duct. Finally, Bowman probe engaged with the tube can be seen over the Hasner's valve. In contrast to the reports of recent researches, the lacrimal probe is inserted into the hole of the prolapsed silicone tube and then into the lacrimal duct, so there is little risk of damaging the tissues of the lacrimal duct.

The advantages of this method are that the patient feels no pain during the making of a hole at the prolapsed silicone tube and the insertion of the lacrimal probe into the hole because the process is taken place at the prolapsed tube. Moreover, the whole process can be performed under topical anesthesia in the outpatient operating room. Some methods for preventing the early prolapse of silicone tube have been presented, including nasal fixation with suture or sponge $[5,6]$. However, due to its complexity, surgery has usually been completed by tying the two free ends of each tube instead of nasal fixation of the silicone tube. The rate of early silicone tube prolapse in children has been reported as $17.5 \%$ to $44 \%$, which has a critical effect on the surgical outcome $[12,13]$.

Opinions may still be divided on whether to reposition the prolapsed silicone tube or insert a new one after removal of prolapsed tube. In our cases, however, the tubes were all prolapsed in a relatively short time after lacrimal intubation due to rubbing by patients, and since there was no change on the tubes to the extent that biofilm was produced, the prolapsed tubes were repositioned without considering re-inserting new tube. So that we didn't need to replace it with a new silicone tube.

The limitation of this method is that while it is feasible for repositioning a dislocated hollow silicone tube after bicanalicular intubation, its use is limited with a solid core silicone tube.

In conclusion, Hole and Lacrimal Probe method is effective for the repositioning of prolapsed silicone tube after bicanalicular intubation of lacrimal system.

\section{Conflict of Interest}

No potential conflict of interest relevant to this article was reported.

\section{References}

1. Park J, Kim J, Baek S. Clinical features and treatment outcomes of patients with tearing after chemotherapy. Eye (Lond) 2019;33:746-53.

2. Fulcher T, O'Connor M, Moriarty P. Nasolacrimal intubation in adults. Br J Ophthalmol 1998;82:1039-41.

3. Baek JS, Lee S, Lee JH, et al. Predictors of silicone tube intubation success in patients with lacrimal drainage system stenosis. Korean J Ophthalmol 2016;30:157-62.

4. Do JR, Kim SH, Chang M. A relationship between the biofilm on silicone tube and outcome of silicone tube intubation in patient with nasolacrimal duct obstruction. Invest Ophthalmol Vis Sci 2014;55:1453.

5. Hakim OM, El-Hag YG. Silicone intubation with trans-sac fixation to prevent tube dislocation. J AAPOS 2005;9:55862.

6. Romano PE. A method of securing double silicone tube intubation of the lacrimonasal system. Arch Ophthalmol 1987;105:746-7.

7. Chu Z, Lu G, Tan Y, Deng Y. Repositioning a prolapsed tube after bicanalicular intubation of the lacrimal system. Ophthalmic Plast Reconstr Surg 2019;35:623-7.

8. He J, Gong J, Zheng Q, Jiang J. Repositioning of the severe prolapsed silicone tubes after bicanalicular nasal intubation: a novel technique. J Ophthalmol 2021;2021:6669717.

9. Brookes JL, Olver JM. Endoscopic endonasal management of prolapsed silicone tubes after dacryocystorhinostomy. Ophthalmology 1999;106:2101-5.

10. Veloudios A, Harvey JT, Philippon M. Long-term placement of silastic nasolacrimal tubes. Ophthalmic Surg 1991;22:225-7.

11. Segal KL, Van Tassel SH, Kim C, et al. Comparison of the extrusion rate of Crawford tubes. Int $J$ Ophthalmol 2015;8:791-3.

12. Cho KW, Lee SY, Kim SJ. Treatment of congenital nasolacrimal duct obstruction using silicone intubation set. $J$ Korean Ophthalmol Soc 1995;36:553-8.

13. Yoon TJ, Na KS, Yoon WJ. The effect of silicone tube intubation in pediatric nasolacrimal duct obstruction. $J$ Korean Ophthalmol Soc 2002;43:155-9. 